\title{
Drosophila fabp is a retinoid-inducible gene required for Rhodopsin-1 homeostasis and photoreceptor survival
}

\author{
Huai-Wei Huang and Hyung Don Ryoo" \\ Department of Cell Biology \\ NYU Grossman School of Medicine \\ 550 First Avenue \\ New York, NY 10016 \\ I Corresponding Author \\ Email: hyungdon.ryoo@nyumc.org
}

Tel: (212) 263-7257

Short Title: fabp regulates Rhodopsin-1 homeostasis

Keywords: Rhodopsin-1, ninaE, fabp, retinal degeneration, Drosophila 


\section{Abstract}

Retinoids act as chromophore co-factors for light-detecting rhodopsin proteins. In vertebrates, retinoids also actively regulate gene expression. Whether retinoids regulate gene expression in Drosophila for a specific biological function remains unclear. Here, we report that Drosophila fatty acid binding protein (fabp) is a retinoid-inducible gene required for Rhodopsin-1 (Rh1) protein homeostasis and photoreceptor survival. Specifically, we performed a photoreceptor-specific gene expression profiling study in flies bearing a misfolding-prone Rhodopsin-1 (Rh1) mutant, nina $E^{G 69 D}$, which serves as a Drosophila model for Retinitis Pigmentosa. nina $E^{G 69 D}$ photoreceptors showed increased expression of genes that control Rh1 protein levels, along with a poorly characterized gene, fabp. We found that in vivo fabp expression was reduced when the retinoids were deprived through independent methods. Conversely, fabp mRNA was induced when we challenged cultured Drosophila cells with retinoic acid. In flies reared under light, loss of fabp caused an accumulation of Rh1 proteins in cytoplasmic vesicles. fabp mutants exhibited light-dependent retinal degeneration, a phenotype also found in other mutants that block light-activated Rh1 degradation. These observations indicate that a retinoid-inducible gene expression program regulates fabp that is required for Rh1 proteostasis and photoreceptor survival. 


\section{Author Summary}

2 Rhodopsins are light-detecting proteins that use retinoids as chromophore co-

3 factors. In vertebrates, retinoids also actively regulate gene expression. Whether

4 retinoids regulate Rhodopsin function aside from its role as a chromophore

5 remains unclear. Here, we report that Drosophila fatty acid binding protein (fabp)

6 is a retinoid-inducible gene required for Rhodopsin-1 (Rh1) protein homeostasis

7 and photoreceptor survival. Specifically, we found that fabp is among the genes

8 induced by a misfolding-prone Rhodopsin-1 (Rh1) mutant, ninaE ${ }^{\mathrm{G} 69 D}$, which

9 serves as a Drosophila model for Retinitis Pigmentosa. We further found that

10 fabp induction in nina $E^{\mathrm{G} 69 D}$ photoreceptors required retinoids. fabp was required

11 in photoreceptors to help degrade light-activated $\mathrm{Rh} 1$. In the absence of fabp,

12 Rh1 accumulated in cytoplasmic vesicles in a light-dependent manner, and

13 exhibited light-dependent retinal degeneration. These observations indicate that

14 a retinoid-inducible gene expression program regulates fabp that is required for

15 Rh1 proteostasis and photoreceptor survival. 


\section{Introduction}

22 Rhodopsins are G-protein coupled proteins associated with retinal chromophores

23 to detect light and initiate signal transduction (1). As in mammals, Drosophila has

24 multiple Rhodopsins, including ninaE (neither inactivation nor afterpotential) that

25 encodes the Rhodopsin-1 (Rh1) protein expressed in R1 to R6 photoreceptors

26 (2-4). Functional Rh1 is covalently attached to the 11-cis-3-hydroxyretinal

27 chromophore, which is derived from dietary vitamin A (5-7). ninaE loss of function

28 results in an impairment of light detection $(4,8)$.

29 Abnormal Rh1 protein homeostasis is a frequent cause of retinal

30 degeneration. One class is caused by a group of ninaE missense mutations that

31 dominantly cause progressive age-related retinal degeneration $(9,10)$. These

32 alleles are analogous to human rhodopsin mutations that underlie age-related

33 retinal degeneration in Autosomal Dominant Retinitis Pigmentosa (ADRP)

34 patients $(11,12)$. Using the Drosophila ninaE ${ }^{G 69 D}$ allele as a model, we

35 previously established that these mutations impose stress in the endoplasmic

36 reticulum (ER), which contributes to retinal degeneration $(13,14)$. The human

37 rhodopsin allele that is most frequently found associated with ADRP, the P23H

38 mutant, similarly causes ER stress in mammalian cells (15).

39 Cellular mechanisms that regulate rhodopsin protein levels affect retinal

40 degeneration. Flies bearing one copy of the nina ${ }^{G 69 D}$ allele have total Rh1

41 protein levels reduced by more than half, indicating that both the mutant and the

42 wild type Rhodopsin-1 proteins undergo degradation in these flies $(9,10)$. Three 
43 ubiquitin ligases that specialize in the degradation of misfolded endoplasmic

44 reticulum (ER) proteins mediate the degradation of Rh1 in ninaE ${ }^{G 69 D}$ flies (16).

45 Overexpression of these ubiquitin ligases can delay the onset of retinal

46 degeneration in Drosophila ninaE ${ }^{G 69 D}$ flies, indicating that excessive misfolded

$47 \mathrm{Rh} 1$ is a contributing factor to retinal degeneration $(14,16)$.

$48 \quad$ Functional wild type Rh1 proteins also undergo degradation after being

49 activated by light. Specifically, this occurs after light-activated Rh1, also referred

50 to as metarhodopsin (M), engages with Arrestin that mediates feedback inhibition

51 (17). Rh1 forms a stable complex with Arrestin and together undergo endocytosis

52 for degradation (18-21). Such degradation of activated Rh1 is essential, as too

53 much Rh1 accumulation in the endosome/lysosome defective photoreceptors

54 results in light-dependent retinal degeneration $(18,22-26)$. In Rh1

55 endocytosis/degradation defective mutants, retinal degeneration could be

56 delayed by conditions that reduce overall Rh1 levels $(24,25,27)$, indicating that

57 too much active Rh1 is a cause of retinal degeneration. These aspects appear to

58 be conserved across phyla, as the human rhodopsin mutants that exhibit high

59 affinities for Arrestin display endosomal abnormalities and are associated with

60 severe forms of $\operatorname{ADRP}(28,29)$.

61 Retinoids are among the molecules implicated in regulating Rh1 protein

62 levels. Deprivation of vitamin A, which serves as a precursor for the retinal

63 chromophore, causes a reduction in overall Rh1 levels (30-34). Such an effect is

64 largely attributed to the importance of chromophores in Rh1 protein maturation.

65 Aside from its role as a rhodopsin cofactor, retinoids regulate gene expression in 
66 vertebrates. The best characterized transcription factors that mediate this

67 response are nuclear hormone receptors, including RAR and RXR, which

68 become transcriptional activators upon binding to all-trans or 9-cis retinoic acid

69 (35). Cellular Retinoic Acid Binding Protein -I and -II (CRABP-1, -II) bind to the

70 lipophilic retinoic acids and deliver them to RAR and RXR in the nucleus $(36,37)$.

71 In addition to mediating the RA signaling response, CRABP-II itself is induced by

72 RA signaling (38). Whether these mechanisms are conserved in Drosophila has

73 not been examined in detail in part because the Drosophila genome does not

74 encode a RAR homolog $(7,39)$. Intriguingly, several Drosophila genes require

75 the retinoid precursor vitamin A for their gene expression $(40,41)$. One such

76 gene is highroad, whose expression is induced by retinoic acids in cultured cells

77 and mediates the degradation $\mathrm{Rh} 1$ in $n i n a E^{G 69 D /+}(42)$. These results suggested

78 that retinoid-mediated gene expression programs may be involved in Rh1

79 homeostasis.

$80 \quad$ Here, we report that Rh1 protein levels are regulated by the Drosophila

81 CRABP homolog, fatty acid binding protein (fabp). Similar to CRABP-II, fabp

82 expression is induced by retinoids. Loss of fabp enhances total Rh1 levels in

83 ninaE wild type and G69D mutant backgrounds. Moreover, loss of fabp causes

84 light-dependent retinal degeneration. Our results indicate that this retinoic acid

85 inducible gene controls Rh1 protein levels, and this regulatory axis is essential

86 for photoreceptor survival.

87

\section{Results}


90 Photoreceptor-specific gene expression profiling shows fabp induction in

\section{1 ninaE ${ }^{G 69 D}$ eyes}

92 To better understand how photoreceptors respond to stress imposed by the

93 nina $E^{G 69 D}$ allele, we performed a photoreceptor-specific gene expression profiling

94 analysis. We specifically employed a previously described approach in which the

95 expression of the nuclear envelope-localized EGFP::Msp300 ${ }^{K A S H}$ is driven in

96 specific cell-types through the Gal4/UAS system to isolate the EGFP-labeled

97 nuclei for RNA-seq analysis $(43,44)$. We used the Rh1-Gal4 driver to isolate

98 ninaE expressing R1 to R6 photoreceptor nuclei from the adult fly ommatidia

99 (Figure 1A). Microscopy imaging confirmed that anti-EGFP beads enriched the

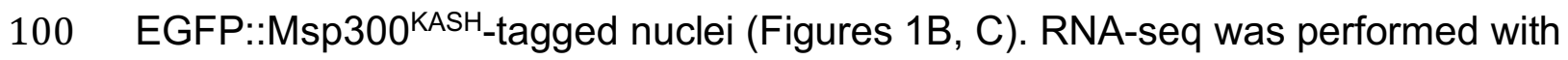

101 nuclei isolated from ninaE wild type and ninaE ${ }^{G 69 D} /+$ photoreceptors.

102 Differential gene expression analysis showed 182 genes whose

103 expression changed with adjusted p values below 0.01 (Supplementary Table 1).

104 Among the most highly induced genes was gstD1 (Figure 1D), which was also

105 identified as an ER stress-inducible gene in a separate study performed with

106 larval imaginal discs (Brown et al., under review). The ER chaperone encoding

$107 c n \times 99 A$ was also induced (Figure 1D), consistent with the previous report that

108 nina $E^{G 69 D}$ imposes ER stress in photoreceptors (13).

109 Also, notable from the differential gene expression analysis was the

110 induction of genes that could affect Rh1 levels. ninaE was itself induced in

111 nina ${ }^{G 69 D}$ samples (Figure 1D). Since nina $E^{G 69 D} /+$ flies have very low Rh1 levels 
$112(9,10)$, we speculate that increases in ninaE transcription may be part of a

113 feedback homeostatic response. Also induced were genes Arrestin 1 (Arr1),

114 Arrestin 2 (Arr2) and culd (Figure 1D), which promote the degradation of light-

115 activated Rh1 in photoreceptors $(17,18,23,45)$.

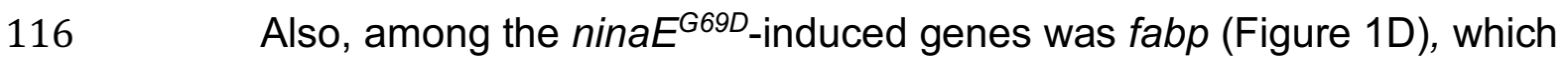

117 encodes a protein homologous to human CRABP-1, -II and FABP5 (Figure 1E).

118 We validated the induction of fabp mRNA in ninaE $E^{G 69} /+$ through $q-R T$ PCR

119 (Figure 1F). The human homologs of fabp are known to bind all trans RA with

120 high affinity $(36,37,46,47)$. Notably, CRABP-II is one of the well-characterized

121 RA inducible genes in mammalian cells (38). fabp drew our interest because a

122 retinoic acid-inducible Drosophila gene, hiro, regulates Rh1 levels in ninaE ${ }^{G 69 D /+}$

123 flies (42).

124

125 fabp expression is regulated by Vitamin A and retinoids

126 To test if Drosophila fabp is also regulated by retinoic acids (RA), we examined

127 fabp mRNA levels through RT-qPCR in Drosophila S2 culture cells treated with or

128 without $10 \mathrm{mM}$ RA. We found that RA treated cells showed an increase in fabp

129 transcripts after 60 minutes of RA exposure (Figure 2A, B).

130 To examine if fabp expression in fly tissues is affected by the availability of

131 Vitamin A and its metabolites, we examined fabp levels in the mutants of ninaD

132 and santa maria that have impaired transport of carotenoids, the precursors of

133 retinoids. These mutants are devoid of retinoids in the retina, as evidenced by

134 defective rhodopsin maturation and light detection $(31,34)$. We found that these 
135 mutants had reduced FABP protein as assessed through western blot of fly head

136 extracts (Figure 2C). Consistently, the mutants also had reduced fabp mRNA

137 levels as assessed through q-RT-PCR (Figure 2D).

138 These observations prompted us to examine if other ninaE ${ }^{G 69 D_{\text {-inducible }}}$

139 genes require santa maria and ninaD for proper expression. We focused on

140 candidates known to be involved in Rh1 protein regulation. Among those tested,

141 the mRNAs of Arr1 and Arr2 were found to be reduced in ninaD or santa maria

142 mutant backgrounds (Figure 2E, F). Not all genes involved in Rh1 homeostasis

143 were affected in these mutants. For example, fatty acid transport protein (fatp) is

144 a gene whose loss-of-function increases Rh1 protein levels (27). fatp mRNA levels

145 were affected neither in the mutant backgrounds of ninaD nor santa maria (Figure

$1462 \mathrm{G})$. These results indicate that the expression of fabp, Arr1, and Arr2 specifically

147 require retinoid and carotenoid transporters, ninaD and santa maria.

149 An fabp protein trap line shows carotenoid-dependent expression in the

\section{$150 \quad$ larval intestine}

151 To independently validate the carotenoid-dependent expression of fabp in vivo, we

152 utilized the fabp protein trap line CA06960. This P-element insertion line has a

153 GFP with splice donor and acceptor sites, designed to make fusion proteins with

154 the endogenous fabp coding sequence (Figure 3A). Anti-GFP western blot of fly

155 extracts confirmed the expression of a GFP-fused protein in adult fly head extracts

156 with the predicted size (Figure 3B). 
157 In the third instar larva, the fabp ${ }^{C A 06960}$ line had GFP expression detectable

158 in several regions of the intestine (Figure 3C, E). Such expression was abolished

159 when the flies were reared in Vitamin A deficient food (Figure 3D). Consistently,

160 the expression of GFP was suppressed in the mutant backgrounds of ninaD and

161 santa maria (Figure $3 F, G$ ). In adult flies, the GFP signal was most prominent in

162 the female abdomen, which was reduced in the ninaD mutant background (Figure

$1633 \mathrm{H}$ ). These results independently support the idea that fabp expression depends

164 on carotenoids.

165

166 Loss of fabp increases Rh1 protein levels

167 To test possible Rh1 regulation by retinoids, we examined several Drosophila

168 homologs of mammalian RA signaling mediators. The candidate genes we

169 analyzed included enzymes that convert Vitamin A to retinoids (e.g. ninaB (48)),

170 nuclear hormone receptors (e.g. knl and eg) and fabp. For our assay, we used

171 nina $E^{G 69 D /+}$ flies, which have drastically reduced Rh1 protein levels as compared

172 to ninaE wild type flies (Figure 4A). Specifically, we drove the expression of RNAi

173 lines that target the genes of interest in the photoreceptors of these $n i n a E^{G 69 D /+}$

174 flies using the Rh1-Gal4/UAS system (Figure 4A). An RNAi line that targeted

175 fabp showed a reproducible effect of partially enhancing Rh1 levels as assessed

176 through western blots of fly head extracts (Figure 4A, B).

177 To validate fabp RNAi results, we employed an fabp loss of function allele,

178 EY02678, which has a P-element inserted near an exon-intron boundary (Figure

$1794 \mathrm{C})$. This allele has strongly reduced FABP expression as assessed through 
180 western blot (Figure 4D). We found that ninaE ${ }^{G 69 D} /+$ Rh1 levels increased in the

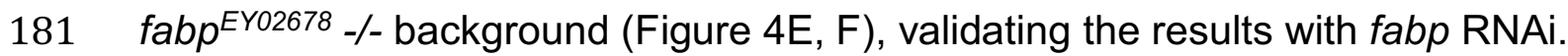

182 We further found that the loss of fabp increased Rh1 levels even in the ninaE

183 wild type flies (Figure 4E, F). When we re-introduced fabp expression in fabp

184 mutant flies using the eye specific GMR-Gal4 driver, Rh1 protein levels were

185 restored to those levels of wild type controls (Figures 4E, F). These results

186 indicate that fabp affects general Rh1 protein levels.

\section{Regulation of Rh1 by fabp is light dependent}

189 Since wild type Rh1 proteins are most notably degraded through light-dependent

190 endocytosis (18-21), we examined whether fabp regulation of Rh1 was light-

191 dependent. We found that fabp mutants showed higher Rh1 levels when the flies

192 were reared under light. Such effect was not seen in flies that were reared in dark

193 (Figure 5A, B).

194 To examine the pattern of Rh1 distribution in photoreceptors, we

195 performed anti-Rh1 immuno-labeling in the adult Drosophila retina. In control wild

196 type flies, Rh1 is predominantly detected in the rhabdomeres of R1 to R6

197 photoreceptor cells organized in a trapezoidal pattern (Figure 5C-E). In fabp -/-

198 flies reared under light, however, there were additional anti-Rh1 signals in

199 intracellular vesicles (Figure 5F).

$200 \quad$ Vesicular Rh1 signals reportedly appear in flies exposed to light,

201 becoming even more prominent in mutants that have defects in Rh1 trafficking to

202 the lysosome $(24,25)$. We found that vesicular Rh1 patterns in fabp -/- eyes were 
203 also light-dependent, as extra-rhabdomeric anti-Rh1 signals mostly disappeared

204 in flies raised under constant darkness (Figure 5G). Together, these results

205 suggest that light-activated Rh1 localize to intracellular vesicles, and these

206 proteins are stabilized in fabp mutants.

208 fabp mutants show light-dependent retinal degeneration that is suppressed

209 in the ninaE $E^{G 69 D} /+$ background

210 To examine whether fabp mutants affect retinal degeneration, we used Rh1-GFP

211 flies with their photoreceptors labeled with green fluorescence. An intact ommatidia

212 has R1-R6 photoreceptors arranged in a trapezoidal pattern that is readily visible

213 as pseudopupils in live flies under low power microscopes (Figure 6A-D). Under

214 standard conditions in which the flies were exposed to moderate light (see

215 Methods), most wild type flies maintained this trapezoidal pattern of Rh1-GFP

216 pseudopupils for the first thirty days after eclosion (Figure 6E, black line).

$217 f a b p^{E Y 02678}$-/- flies, on the other hand, showed signs of severe age-related retinal

218 degeneration under otherwise identical conditions: Specifically, a few flies of this

219 genotype began showing the loss of Rh1-GFP pseudopupils at day 15, with almost

220 all examined flies having signs of retinal degeneration by day 28 (Figure 6E, red

221 line; 6F). The difference between wild type controls and fabp YYO2678 $_{-/-}$was

222 statistically significant (Log-rank test, $\mathrm{p}<0.0001)$. Retinal degeneration in fabp

223 mutants was light-dependent, as those reared in the dark did not exhibit signs of

224 photoreceptor degeneration (Figure 6G). The light-dependent nature of

225 photoreceptor degeneration correlated with fabp's effect on Rh1 levels. 
226 As reported previously, ninaE $6690 /+$ flies showed age-related retinal 227 degeneration that started occurring around day 17 , with most flies exhibiting retinal

228 degeneration at day 30 (Figure 6E, black dotted line). Surprisingly, flies containing 229 nina $E^{G 69 D /+}$ in the fabp -/- background had a significantly delayed course of retinal 230 degeneration, with most flies still showing intact Rh1-GFP pseudopupils 30 days 231 after eclosion. While surprising, such genetic interaction with nina $E^{G 69 D}$ is not 232 unprecedented. Previous studies found that mutants that increase wild type Rh1 233 levels, such as fatty acid transport protein (fatp), cause severe retinal degeneration.

234 Such retinal degeneration is suppressed in the ninaE $E^{G 69 D} /+$ background (27).

\section{Discussion}

237 The biological role of retinoic acid signaling is now well-delineated in vertebrates.

238 However, the biological role and the mechanism of retinoid-mediated gene 239 expression in Drosophila have remained unclear. Here, we showed that the 240 expression of Drosophila fabp is regulated by vitamin A and retinoids. We found 241 that fabp regulates Rh1 protein levels and loss of fabp results in retinal 242 degeneration.

243 In Drosophila, the major phenotype associated with vitamin A deficiency is 244 the loss of visual function. Significantly lower levels of Rh1 protein are detected 245 under these conditions, as vitamin A deficiency would deplete the chromophore 246 11-cis 3-hydroxy retinal, which is normally required for proper Rh1 maturation. Our 247 data presented here indicates that there is an additional layer of Rh1 regulation 248 through retinoid-inducible fabp. 
250 involved in the endosomal/lysosomal degradation of light-activated Rh1.

251 Specifically, we found that Rh1 levels increase in fabp mutants when flies were

252 reared under light, but not when the flies were reared in constant darkness.

253 Furthermore, immunohistochemical analysis shows that Rh1 accumulates in

254 intracellular vesicles of fabp mutants only when the flies were reared under light.

255 Since it is now well-documented that light-activated Rh1 undergoes endocytosis

256 and lysosomal degradation (18-21), we interpret that fabp is specifically involved

257 in this process.

258 We further note that the accelerated retinal degeneration phenotype of fabp

259 mutants are reminiscent of other genetic conditions that result in endosomal

260 accumulation of Rh1 in response to light. Examples of this type include mutations

261 in norpA, culd, retromer complex proteins, and fatty acid transport protein $(18,25$,

262 27, 45). As these genes normally regulate light-dependent internalization of Rh1,

263 it is likely that excessive levels of light-activated Rh1 is contributing to the retinal

264 degeneration phenotype under these conditions.

265 If it is indeed the excessive Rh1 levels in fabp mutants that are the cause

266 of retinal degeneration, reduction of $\mathrm{Rh} 1$ in these flies would delay retinal

267 degeneration. This is what we observed when we examined the fabp phenotype in

268 the nina $E^{G 69 D /+}$ background, which drastically reduces overall Rh1 levels. The way

269 fabp and ninaE ${ }^{G 69 D}$ genetically interacted with each other was interesting in that

270 ninaE G69D/+ flies normally show age-related retinal degeneration, which was also

271 delayed in the fabp mutant background. This hints at the possibility that too low 
272 Rh1 levels is a contributing factor of retinal degeneration in ninaE ${ }^{G 69 D /+}$

273 photoreceptors. Partial restoration functional Rh1 in the fabp -/- background

274 appears to help delay retinal degeneration in $n i n a E^{G 69 D} /+$ eyes.

275 In conclusion, we showed that fabp expression is regulated by retinoids and

276 carotenoids in Drosophila. Our results indicate that retinoids not only serve as

277 chromophores for rhodopsins, but have additional roles in regulating Rh1 protein

278 levels. It remains to be examined whether mammalian CRAPBs similarly regulate

279 rhodopsin levels and affect retinal degeneration in response to retinoic acids.

280

281 Acknowledgements

282 We thank Drs. Vikke Weake, Jason Gerstner for fly lines and antibodies. This work 283 was supported by the NIH grant R01 EY020866 to H.D.R.

285 Author contributions: H.H. performed all experiments. H.H. and H.D.R. together 286 designed experiments and analyzed data. H.D.R. wrote the manuscript draft with 287 H.H.'s edits and inputs.

289 Competing interests: The authors declare no competing interests.

291 Materials and Methods

292

\section{Fly Genetics}


294 All fly crosses were maintained in $25^{\circ} \mathrm{C}$. Unless otherwise stated, flies were

295 reared with a standard cornmeal-agar diet supplemented with molasses. Vitamin

296 A deficient food was made by mixing $12 \mathrm{~g}$ yeast, $1.5 \mathrm{~g}$ agar, $7.5 \mathrm{~g}$ sucrose, 30

$297 \mathrm{mg}$ cholesterol, $3.75 \mathrm{ml}$ of $1.15 \mathrm{M}$ Nippagin, $720 \mu \mathrm{l}$ propionic acid in distilled water

298 volume of $150 \mathrm{ml}$.

299 Uas-fabp had EGFP fused in frame with the fabp's N-terminal coding

300 sequence. EGFP-fabp was subcloned into the pUAST plasmid, and the resulting

301 construct was injected by Best Gene, Inc., to generate the uas-fabp transgenic

302 line.

303 We used the following flies that had been reported previously: Rh1-Gal4

304 (49), Rh1-GFP (50), ninaE ${ }^{G 69 D}(9)$, santa maria ${ }^{1}(34)$, ninaD ${ }^{1}(31)$, uas-dicer2

305 (51), UAS-EGFP::Msp-300KASH (44). fabp ${ }^{C A 06960}$ (52) and fabp ${ }^{E Y 02678}$ were

306 obtained from the Bloomington Drosophila Stock Center (stock numbers \#50808

307 and \#15579, respectively).

308 The RNAi lines used are as follows: uas-lacZ RNAi (53), uas-fabp RNAi

309 (Bloomington Stock Center \# 34685), uas-fatp RNAi (Bloomington Stock Center

310 \# 55273), uas-ninaB RNAi (Bloomington Stock Center \#34994), uas-knrl RNAi

311 (Bloomington Stock Center \# 36664), uas-eg RNAi (Bloomington \# 35234).

312 These lines were crossed to the female virgins of the genotype: Rh1-Gal4;

313 ninaE ${ }^{G 69 D} / T M 6 B$. We collected non-TM6B progeny of these crosses to examine

314 Rh1 protein and RNA.

315

316 Photoreceptor-specific nuclear RNA extraction 
317 We followed a published protocol to isolate Rh1-Gal4>UAS-EGFP::Msp-300KASH_

318 positive nuclei (44). In brief, approximately 500 adult fly heads (from flies within 5

319 days of eclosion) per genotype were lysed in ice-cold nuclear isolation buffer (10

320 mM HEPES-KOH, $\mathrm{pH}$ 7.5; $2.5 \mathrm{mM} \mathrm{MgCl}_{2} ; 10 \mathrm{mM} \mathrm{KCl)} \mathrm{with} \mathrm{a} \mathrm{dounce}$

321 homogenizer. The homogenate was filtered through a $40 \mu \mathrm{m}$ Flowmi cell strainer

322 (WVR, cat. \#BAH136800040), and the filtrate was incubated with anti-EGFP-

323 coupled protein G Dynabeads (Invitrogen, cat. \#10003D) for 1 hour at $4^{\circ} \mathrm{C}$. The

324 beads were collected using a magnetic microcentrifuge tube holder (Sigma, cat.

325 \#Z740155). Following washes with wash buffer (PBS, $\mathrm{pH} 7.4 ; 2.5 \mathrm{mM} \mathrm{MgCl}$ ), the

326 beads were resuspended in a final volume of $150 \mu \mathrm{L}$ of wash buffer. Then the

327 post-isolation nuclei were suspended in $1 \mathrm{~mL}$ of Trizol reagent (Life Technologies,

328 cat. \#15596018) for RNA extraction following standard procedures. Prior to RNA

329 precipitation with isopropanol, 0.3M sodium acetate and glycogen were added to

330 facilitate visualization of the RNA pellet. We then suspended the pellet in RNAse-

331 free water and purified it using a Qiagen RNeasy MinElute cleanup kit (Qiagen,

332 cat. \#74204) following standard protocols.

334 Preparation of cDNA libraries, RNA-seq and data processing

335 The NYU Genome Technology Center performed library preparation and RNA

336 sequencing. We quantified RNA on an Agilent 2100 BioAnalyzer (Agilent, cat.

337 \#G2939BA). For cDNA library preparation and ribodepletion, we utilized a

338 custom Drosophila Nugen Ovation Trio low-input library preparation kit (Tecan 
339 Genomics), using approximately $20 \mathrm{ng}$ total RNA per sample. For sequencing,

340 we performed paired-end 50bp sequencing of samples on an Illumina NovaSeq

3416000 platform (Illumina, cat. \#20012850) using half of a 100 cycle SP flow cell

342 (Illumina, cat. \#20027464). We used the bcl2fastq2 Conversion software (v2.20)

343 to convert per-cycle BCL base call files outputted by the sequencing instrument

344 (RTA v3.4.4) into the fastq format in order to generate per-read per-sample fastq

345 files. For subsequent data processing steps, we used the Seq-N-Slide automated

346 workflow developed by Igor Dolgalev (https://github.com/igordot/sns). For read

347 mapping, we used the alignment program STAR (v2.6.1d) to map reads of each

348 sample to the Drosophila melanogaster reference genome dm6, and for quality

349 control we used the application Fastq Screen (v0.13.0) to check for

350 contaminating sequences. We employed featureCounts (Subread package

351 v1.6.3) to generate matrices of read counts for annotated genomic features. For

352 differential gene statistical comparisons between groups of samples contrasted

353 by genotype, we used the DESeq2 package (R v3.6.1) in the R statistical

354 programming environment. We excluded genes with baseMean counts less than

355300 so as to avoid artifacts due to varying extent of nuclei purification.

356

357 Immunofluorescence and Western Blots

358 We followed standard protocols for western blots and whole mount immuno-

359 labeling experiments using the following primary antibodies: Mouse monoclonal

360 4C5 anti-Rh1 (Developmental Studies Hybridoma Bank, used at 1:5000 for 
361 western blots), anti- $\beta$ tubulin antibody (Covance \#MMS-410P), Rabbit anti-GFP

362 (Invitrogen \#A-6455), anti-FABP antibody (54).

363

\section{$364 \quad$ RT-PCR}

365 We performed qRT-PCR using Power SYBR green master mix kit (Thermo

366 Fisher). The primer sequences are as follows:

367 Rpl15F: AGGATGCACTTATGGCAAGC

368 Rpl15R: GCGCAATCCAATACGAGTTC

369 FatpF: CTCCCGGTGAGTGCAATAGCTT

370 FatpR: GCGGTGTGGTACAAAGGCAA

371 Arr1F: CATGAACAGGCGTGATTTTGTAG

372 Arr1R: TTCTGGCGCACGTACTCATC

373 Arr2F: TCGATGGAGTGATTGTGGTGG

374 Arr2R: GCGACCATAGCGATAGGTGG

375 Fabp-1F: CCGAGGTCTCAGTGTGCTC

376 Fabp-1R: CCGAGGTCTCAGTGTGCTC

377 Fabp-2F: CACAGTGGAGGTGACCTTGG

378 Fabp-2R: GATGCTCTTGACGTTGCGAC

379 TubF: CTCAGTGCTCGATGTTGTCC

380 TubR: GCCAAGGGAGTGTGTGAGTT

381

382 Retinal degeneration assay 
383 We performed all retinal degeneration assays in the $c n, b w-/-$ background to

384 eliminate eye pigments that otherwise affect the course of retinal degeneration.

385 The flies were incubated in the $25^{\circ} \mathrm{C}$ incubator with 1000 lux of light. For retinal

386 degeneration assays under constant darkness, the flies were reared in an

387 enclosed cardboard box in the $25^{\circ} \mathrm{C}$ incubator. Retinal degeneration was

388 assessed based on green fluorescent pseudopupils originating from the Rh1-

389 GFP transgene. We interpreted clear trapezoidal pseudopupils as evidence in

390 intact photoreceptors, while its disappearance was construed as a sign of retinal

391 degeneration. The number of flies analyzed for each genotype in Figure 6E is as

392 follows:

393 wild type, 48 flies; fabp ${ }^{E Y 02678}, 52$ flies; ninaE ${ }^{G 69 D} /+, 50$ flies; ninaE ${ }^{G 69 D}$,

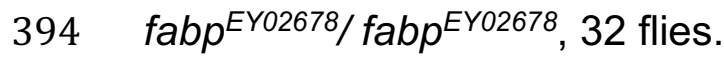

395 For Figures 6F and G, 50 flies were analyzed for each genotype.

397 Quantification and statistics

398 To quantify proteins in gels, we measured average pixel intensities of western

399 blot bands using Image J, and normalized them to anti- $\beta$ tubulin bands. Graphs

400 were generated after at least three independent measurements and $p$ values

401 were calculated using a paired t-test. For retinal degeneration assays, we used

402 the Log-rank (Mantel-Cox) text. Graphs were made using the Graphpad Prism

403 program. All error bars represent SEM (Standard error of the mean). 


\section{References}

405 1. Yau KW, Hardie, R.C. Phototransduction motifs and variations. Cell.

$406 \quad 2009 ; 139(2): 246-64$.

407 2. O'Tousa JE, Baehr, W., Martin, R.L., Hirsh, J., Pak, W.L., Applebury, M.L.

408 The Drosophila ninaE gene encodes an opsin. Cell. 1985;40(4):877-82.

409 3. Zuker CS, Cowman, A.F., Rubin, G.M. Isolation and structure of a

410 rhodopsin gene from D. melanogaster. Cell. 1985;40(4):851-8.

411 4. Montell C. Drosophila visual transduction. Trends Neurosci.

$412 \quad 2012 ; 35(6): 356-63$.

413 5. Nichols R, Pak WL. Characterization of Drosophila melanogaster

414 rhodopsin. J Biol Chem. 1985;260(23):12670-4.

415 6. Seki T, Isono K, Ito M, Katsuta Y. Flies in the group Cyclorrhapha use

416 (3S)-3-hydroxyretinal as a unique visual pigment chromophore. Eur J Biochem.

$417 \quad$ 1994;226(2):691-6.

418 7. Dewett D, Lam-Kamath K, Poupault C, Khurana H, Rister J. Mechanisms

419 of vitamin A metabolism and deficiency in the mammalian and fly visual system.

420 Dev Biol. 2021.

421 8. Scavarda NJ, O'Tousa, J., Pak, W.L. Drosophila locus with gene-dosage

422 effects on rhodopsin. Proc Natl Acad Sci USA. 1983;80(14):4441-5.

423 9. Colley NJ, Cassill, J.A., Baker, E.K., Zuker, C.S. Defective intracellular

424 transport is the molecular basis of rhodopsin-dependent dominant retinal

425 degeneration. Proc Natl Acad Sci USA. 1995;92(7):3070-4. 
426 10. Kurada P, O'Tousa, J.E. Retinal degeneration caused by dominant

427 rhodopsin mutations in Drosophila. Neuron. 1995;14(3):571-9.

428 11. Dryja T, McGee, T.L., Reichel, E., Hahn, L.B., Cowley, G.S., Yandell,

429 D.W., Sandberg, M.A., Berson, E.L. A point mutation of the rhodopsin gene in

430 one form of retinitis pigmentosa. Nature. 1990;343(6256):364-6.

431 12. Sung CH, Davenport, C.M., Hennessey, J.C., Maumenee, I.H., Jacobson,

432 S.G., Heckenlively, J.R., Nowakowski, R., Fishman, G., Gouras, P., Nathans, J.

433 Rhdopsin mutations in autosomal dominant retinitis pigmentosa. Proc Natl Acad

434 Sci USA. 1991;88(15):6481-5.

435 13. Ryoo HD, Domingos PM, Kang MJ, Steller H. Unfolded protein response

436 in a Drosophila model for retinal degeneration. Embo j. 2007;26(1):242-52.

437 14. Kang M-J, Ryoo, H.D. Suppression of retinal degeneration in Drosophila

438 by stimulation of ER-Associated Degradation. Proc Natl Acad Sci USA.

439 2009;106(40):17043-8.

440 15. Lin JH, Li, H., Yasumura, D., Cohen, H.R., Zhang, C., Panning, B.,

441 Shokat, K.M., Lavail, M.M., Walter, P. IRE1 signaling affects cell fate during the

442 unfolded protein response. Science. 2007;318(5852):944-9.

443 16. Xu J, Zhao H, Wang T. Suppression of retinal degeneration by two novel

444 ERAD ubiquitin E3 ligases SORDD1/2 in Drosophila. PLoS Genet.

$445 \quad 2020 ; 16(11): e 1009172$.

446 17. Dolph PJ, Ranganathan R, Colley NJ, Hardy RW, Socolich M, Zuker CS.

447 Arrestin function in inactivation of $\mathrm{G}$ protein-coupled receptor rhodopsin in vivo.

448 Science. 1993;260(5116):1910-6. 
449 18. Alloway PG, Howard L, Dolph PJ. The formation of stable rhodopsin-

450 arrestin complexes induces apoptosis and photoreceptor cell degeneration.

$451 \quad$ Neuron. 2000;28(1):129-38.

452 19. Orem NR, Dolph, P.J. Epitope masking of rhabdomeric rhodopsin during

453 endocytosis-induced retinal degeneration. Mol Vis. 2002;8:455-61.

454 20. Satoh AK, Ready DF. Arrestin1 mediates light-dependent rhodopsin

455 endocytosis and cell survival. Curr Biol. 2005;15(19):1722-33.

456 21. Satoh AK, Xia H, Yan L, Liu CH, Hardie RC, Ready DF. Arrestin

457 translocation is stoichiometric to rhodopsin isomerization and accelerated by

458 phototransduction in Drosophila photoreceptors. Neuron. 2010;67(6):997-1008.

459 22. Xu H, Lee SJ, Suzuki E, Dugan KD, Stoddard A, Li HS, et al. A lysosomal

460 tetraspanin associated with retinal degeneration identified via a genome-wide

461 screen. Embo j. 2004;23(4):811-22.

462 23. Orem NR, Xia L, Dolph PJ. An essential role for endocytosis of rhodopsin

463 through interaction of visual arrestin with the AP-2 adaptor. J Cell Sci.

$464 \quad 2006 ; 119(P t$ 15):3141-8.

465 24. Chincore Y, Mitra, A., Dolph, P.J. Accumulation of rhodopsin in late

466 endosomes triggers photoreceptor cell degeneration. PLoS Genet.

$467 \quad 2009 ; 5(2): e 1000377$.

468 25. Wang S, Tan, K.L., Agosto, M.A., Xiong, B., Yamamoto, S., Sandoval, H., 469 Jaiswal, M., Bayat, V., Zhang, K., Charng, W.L., David, G., Duraine, L.,

470 Venkatachalam, K., Wensel, T.G., Bellen, H.J. The retromer complex is required 
471 for rhodopsin recycling and its loss leads to photoreceptor degeneration. PLoS

472 Biology. 2014;12(4):e1001847.

473 26. Hebbar S, Lehmann M, Behrens S, Hälsig C, Leng W, Yuan M, et al.

474 Mutations in the splicing regulator Prp31 lead to retinal degeneration in

475 Drosophila. Biol Open. 2021;10(1).

476 27. Dourlen P, Bertin B, Chatelain G, Robin M, Napoletano F, Roux MJ, et al.

477 Drosophila fatty acid transport protein regulates rhodopsin- 1 metabolism and is

478 required for photoreceptor neuron survival. PLoS Genet. 2012;8(7):e1002833.

479 28. Chuang JZ, Vega C, Jun W, Sung CH. Structural and functional

480 impairment of endocytic pathways by retinitis pigmentosa mutant rhodopsin-

481 arrestin complexes. J Clin Invest. 2004;114(1):131-40.

482 29. Chen J, Shi G, Concepcion FA, Xie G, Oprian D, Chen J. Stable

483 rhodopsin/arrestin complex leads to retinal degeneration in a transgenic mouse

484 model of autosomal dominant retinitis pigmentosa. J Neurosci.

485 2006;26(46):11929-37.

486 30. Harris WA, Ready, D.F., Lipson, E.D., Hudspeth, A.J., Stark, W.S. Vitamin

487 A deprivation and Drosophila photopigments. Nature. 1977;266(5603):648-50.

488 31. Gu G, Yang, J., Mitchell, K.A., O'Tousa, J.E. Drosophila ninaB and ninaD

489 act outside of retina to produce rhodopsin chromophore. J Biol Chem.

$490 \quad 2004 ; 279(18): 18608-13$.

491 32. Wang T, Montell, C. Rhodopsin formation in Drosophila is dependent on

492 the PINTA retinoid-binding protein. J Neurosci. 2005;25(21):5187-94. 
493 33. Ahmad ST, Joyce, M.V., Boggess, B., O'Tousa, J.E. The role of

494 Drosophila ninaG oxidoreductase in visual pigment chromophore biogenesis. J

495 Biol Chem. 2006;281(14):9205-9.

496 34. Wang T, Jiao, Y., Montell, C. Dissection of the pathway required for

497 generation of vitamin A and for Drosophila phototransduction. J Cell Biol.

$498 \quad 2007 ; 177(2): 305-16$.

499 35. Mangelsdorf DJ, Evans, R.M. The RXR heterodimers and orphan

500 receptors. Cell. 1995;83(6):841-50.

501 36. Kleywegt GJ, Bergfors, T., Senn, H., Le Motte, P., Gsell, B., Shudo, K.,

502 Jones, T.A. Crystal structures of cellular retinoic acid binding proteins I and II in

503 complex with all-trans-retinoic acid and a synthetic retinoid. Structure.

$504 \quad 1994 ; 2(12): 1241-58$.

505 37. Napoli JL. Cellular retinoid binding-proteins, CRBP, CRABP, FABP5:

506 Effects on retinoid metabolism, function and related diseases. Pharmacol Ther.

$507 \quad 2017 ; 173: 19-33$.

508 38. Durand B, Saunders, M., Leroy, P., Leid, M., Chambon, P. All-trans and 9-

509 cis retinoic acid induction of CRABPII transcription is mediated by RAR-RXR

510 heterodimers bound to DR1 and DR2 repeated motifs. Cell. 1992;71(1):73-85.

511 39. King-Jones K, Thummel, C.S. Nuclear receptors-a perspective from

512 Drosophila. Nat Rev Genet. 2005;6(4):311-23.

513 40. Picking WL, Chen, D.M., Lee, R.D., Vogt, M.E., Polizzi, J.L., Marietta,

514 R.G., Stark, W.S. Control of Drosophila opsin gene expression by carotenoids 
515 and retinoic acid: northern and western analysis. Exp Eye Res. 1996;63(5):493-

516500.

517 41. Shim K, Picking, W.L, Kutty, R.K., Thomas, C.F., Wiggert, B.N., Stark,

518 W.S. Control of Drosophila retinoid and fatty acid binding glycoprotein expression

519 by retinoids and retinoic acid: northern, western and immunocytochemical

520 analysis. Exp Eye Res. 1997;65(5):717-27.

521 42. Huang HW, Brown, B., Chung, J., Domingos, P.M., Ryoo, H.D. highroad is

522 a carboxypeptidase induced by retinoids to clear mutant Rhodopsin-1 in

523 Drosophila Retinitis Pigmentosa models. Cell Rep. 2018;22(6):1384-91.

524 43. Hall H, Medina P, Cooper DA, Escobedo SE, Rounds J, Brennan KJ, et al.

525 Transcriptome profiling of aging Drosophila photoreceptors reveals gene

526 expression trends that correlate with visual senescence. BMC Genomics.

$527 \quad 2017 ; 18(1): 894$.

528 44. Ma J, Weake VM. Affinity-based isolation of tagged nuclei from Drosophila

529 tissues for gene expression analysis. J Vis Exp. 2014(85).

530 45. $\mathrm{Xu} \mathrm{Y,} \mathrm{Wang} \mathrm{T.} \mathrm{CULD} \mathrm{is} \mathrm{required} \mathrm{for} \mathrm{rhodopsin} \mathrm{and} \mathrm{TRPL} \mathrm{channel}$

531 endocytic trafficking and survival of photoreceptor cells. J Cell Sci.

$532 \quad 2016 ; 129(2): 394-405$.

533 46. Giguere V, Lyn, S., Yip, P., Siu, C.H., Amin, S. Molecular cloning of cDNA

534 encoding a second cellular retinoic acid-binding protein. Proc Natl Acad Sci USA.

535 1990;87(16):6233-7. 
536 47. Shaw N, Elholm, M., Noy, N. Retinoic acid is a high affinity selective ligand

537 for the peroxisome proliferator-activated receptor beta/delta. J Biol Chem.

$538 \quad 2003 ; 278(43): 41589-92$.

539 48. von Lintig J, Dreher, A., Kiefer, C., Wernet, M.F., Vogt, K. Analysis of the

540 blind Drosophila mutant ninaB identifies the gene encoding the key enzyme for

541 vitamin A formation invivo. Proc Natl Acad Sci USA. 2001;98(3):1130-5.

542 49. Mollereau B, Wernet MF, Beaufils P, Killian D, Pichaud F, Kuhnlein R, et

543 al. A green fluorescent protein enhancer trap screen in Drosophila photoreceptor

544 cells. Mech Dev. 2000;93(1-2):151-60.

545 50. Pichaud F, Desplan C. A new visualization approach for identifying

546 mutations that affect differentiation and organization of the Drosophila ommatidia.

547 Development. 2001;128(6):815-26.

548 51. Dietzl G, Chen, D., Schnorrer, F., Su, K.C., Barinova, Y., Fellner, M.,

549 Gaser, B., Kinsey, K., Oppel, S., Scheiblauer, S., Couto, A., Marra, V., Keleman,

550 K., Dickson, B.J. A genome-wide transgenic RNAi library for conditional gene

551 inactivation in Drosophila. Nature. 2007;448(7150):151-6.

552 52. Buszczak M, Paterno, S., Lighthouse, D., Bachman, J., Planck, J., Owen,

553 S., Skora, A.D., Nystul, T.G., Ohlstein, B., Allen, A., Wilhelm, J.E., Murphy, T.D.,

554 Levis, R.W., Matunis, E., Srivali, N., Hoskins, R.A., Spradling, A.C. The Carnegie

555 protein trap library: A versatile tool for Drosophila development studies. Genetics.

$556 \quad 2007 ; 175(3): 1505-31$.

557 53. Kennerdell JR, Carthew RW. Heritable gene silencing in Drosophila using 558 double-stranded RNA. Nat Biotechnol. 2000;18(8):896-8. 
559 54. Gerstner JR, Vanderheyden WM, Shaw PJ, Landry CF, Yin JC. Fatty-acid

560 binding proteins modulate sleep and enhance long-term memory consolidation in

561 Drosophila. PLoS One. 2011;6(1):e15890.

562

563

$564 \quad$ Figure legends

565

566 Figure 1: Photoreceptor-specific gene expression profiling in nina $E^{G 69 D}$ eyes.

567 (A) A schematic diagram of the Drosophila ommatidium. Shown are seven

568 photoreceptor cells, R1 to R7. KASH-GFP (green) coats the outer membranes of

569 R1 to R6 nuclei (red). Rh1 localizes to the apical membrane structure known as

570 rhabdomeres (gray). (B, C) Purification of photoreceptor nuclei tagged with EGFP-

571 Msp300 KASH (KASH-GFP). Rh1-Gal4 was used to express uas-KASH-GFP in R1

572 to R6 photoreceptors. Nuclei are labeled with DAPI (red) and the anti-GFP beads

573 are in green. (B) Before anti-GFP bead purification. (C) After purification, the DAPI

574 labeled nuclei are associated with the beads. (D) Volcano plot of differential gene

575 expression compared between ninaE wild type and ninaE ${ }^{G 69 D /+}$ photoreceptors.

576 The $y$ axis shows $-\log _{10}$ (adjusted $p$ value). The $x$ axis represents $\log _{2}$ fold change,

577 with those whose expressions increase in ninaE $E^{G 9 D} /+$ on the right (adjusted

$578 p<0.01$ are labeled in red). $\log _{2}$ fold change above 2.5 is not in scale. Genes with

579 nonsignificant changes (adjusted $p>0.01$ ) are in gray. (E) Sequence comparison

580 between Drosophila FABP, human CRABP-II and FABP5. (F) qRT-PCR results of

581 fabp from ninaE wild type (left) and ninaE $E^{G 69 D} /+$ fly heads. Error bars represent 
582 Standard Error (SE). t-test was used to assess statistical significance. ${ }^{*}=p<0.05$.

583

584 Figure 2: fabp expression is regulated by carotenoids and retinoic acid. (A)

585 The time course of fabp mRNA induction as assessed through semi-quantitative

586 RT-PCR for fabp (top gel) and the control Rp/15 (bottom gel). Cultured Drosophila

587 S2 cells were treated with either control DMSO (left 4 lanes) or with 10mM all-trans

588 retinoic acid (RA, right 4 lanes) for the indicated periods of time. (B) qRT-PCR of

589 fabp from S2 cells treated with DMSO (black) or 10mM all-trans retinoic acid (grey

590 bars) for the indicated period of time. The y axis shows fold induction as compared

591 to the results from the DMSO controls. (C) Western blot of FABP (top gel) and $\beta$ -

592 Tubulin (bottom gel) from adult fly head extracts of the indicated genotypes. $w^{1118}$

593 flies were used as wild type controls. (D-G) qRT-PCR-based assessment of

594 indicated mRNAs from adult fly heads of the indicated genotypes. The levels of

595 fabp (D), Arr1 (E), Arr2 (F), fatp (G) are shown. The y axis shows fold changes

596 compared to results obtained from wild type control samples. In all qRT-PCRs,

597 RpL15 qRT-PCR results were used to normalize the levels of transcripts of

598 interest. Error bars represent standard error (SE). Statistical significance was

599 assessed through two tailed t-tests. ${ }^{* *}=p<0.005,{ }^{* * *}=p<0.0005,{ }^{* * * *}=p<0.0001$.

600

601 Figure 3: The expression of an fabp GFP protein trap line requires Vitamin A

602 and its transporter genes. (A) The structure of the fabp locus and the CA06960

603 protein trap line. (B) Anti-GFP western of adult head extracts from control and the

604 CA06960 protein trap line. (C-G) Images of dissected fabp $p^{C A 06960}$ third instar larval 
605 intestines immuno-labeled with anti-GFP antibody (green). GFP signal is detected

606 in distinct regions of the intestine in flies reared under standard conditions (C),

607 which decreases in those reared in a Vitamin A deficient food (D). (E-G) GFP signal

608 from flies reared under standard food in the control genotype (E), in the ninaD ${ }^{1}$

609 mutants (F) and in the santa maria ${ }^{1}$ mutant background (G). (H) GFP signal of

610 fabp ${ }^{C A 06960}$ adult females in the control genetic background (left two flies) and in

611 the $\operatorname{nina} D^{1}$ mutant background (right two flies). The scale bar in $\mathrm{C}$ is for images $\mathrm{C}$ -

612 G.

613

614 Figure 4: loss of fabp enhances Rhodopsin-1 protein levels. (A, B) Western

615 blot of Rhodopsin-1 (Rh1) and $\beta$-tubulin from fly heads extracts. (A) The first lane

616 is from ninaE wild type samples. The remaining lanes are from nina ${ }^{G 69 D /+}$ flies

617 with the indicated genes knocked down through RNAi with the Rh1-Gal4 driver.

618 lacZ RNAi (lane 2) was used as a negative control. (B) Quantification of relative

619 Rh1 band intensities as normalized to $\beta$-tubulin. (C) A schematic diagram of the

620 fabp locus and the EY02678 P-element insertion site. (D) Western blot of FABP

621 and $\beta$-tubulin from adult fly head extracts of wild type or fabp $p^{E Y 02678}$ flies. (E)

622 Western blot of Rh1 in flies from the head extracts of the indicated genotypes. fabp

623 wild type (lane 1), fabp EY02678 mutants (lane 2), fabp $^{E Y 02678}$ mutants rescued with

624 GMR-Gal4 driven uas-fabp expression (lane 3). Lanes 4 -6 show anti-Rh1 blots in

625 the ninaE ${ }^{G 69 D} /+$ background, with fabp wild type (lane 4), fabp ${ }^{E Y 0678}$ (lane 5), and

626 fabp ${ }^{E Y 02678}$ mutants rescued with GMR-Gal4/uas-fabp (lane 6). (F) Quantification

627 of relative Rh1 band intensities as normalized with $\beta$-tubulin. Error bars represent 
628 standard error (SE). Statistical significance was assessed through t-test. * $=$

$629 p<0.05,{ }^{* \star}=p<0.005$

630

631 Figure 5: Rh1 regulation by fabp is light-dependent. (A) An anti-Rh1 western

632 blot of control fabp wild type (lanes 1, 3) or fabp EY02678 -/- (lanes 2, 4) fly head

633 extracts. The flies were either reared under constant light (lanes 1, 2) or in constant

634 darkness (lanes 3,4). The lower band shows an anti-Tubulin blot as a control. (B)

635 Quantification of anti-Rh1 blot intensities, normalized to anti-Tubulin blots.

636 Statistical significance was assessed through t-test. * $=p<0.05$. (C) A schematic

637 diagram of adult Drosophila ommatidia, with rhabdomeres labeled as black circles,

638 and endosomal vesicles as white circles. (D-G) Anti-Rh1 labeling of adult

639 Drosophila ommatidia. (D, E) Control fabp wild type eyes. (F, G) fabp EY02678 -/-

640 eyes. Fly samples (D, F) were reared under constant light before being processed

641 for fixation and immuno-labeling. By contrast, samples (E, G) were reared under

642 constant darkness before processing. The scale bar in $G$ applies for images D -

643 G.

644

645 Figure 6: Retinal degeneration in fabp loss of function mutants. (A-D)

646 Representative images of adult fly eyes of the indicated genotypes at 14 days after

647 eclosion. White arrows point to the trapezoidal pattern of Rh1-GFP pseudopupils,

648 which are indicative of intact photoreceptors. (E) The course of retinal

649 degeneration in flies of the indicated genotypes, assessed through Rh1-GFP

650 pseudopupils. Flies were reared under 1000 lux constant light. The y axis shows 
$651 \%$ of flies with intact photoreceptors. The $\mathrm{x}$ axis indicates the days after eclosion.

652 fabp ${ }^{\text {YY02678 }}$ mutant flies had accelerated retinal degeneration as compared to

653 control wild type flies, with $p<0.0001$ (Log-rank test). (F, G) Comparison of retinal

654 degeneration state at 19 and 21 days after eclosion in flies reared under constant

655 light $(F)$, or under constant darkness $(G)$. The y axis shows fraction of flies with

656 intact photoreceptors. Black bars represent fabp wild type control flies, and the

657 gray bars indicate fabp ${ }^{E Y 020866}$-/- flies.

658 

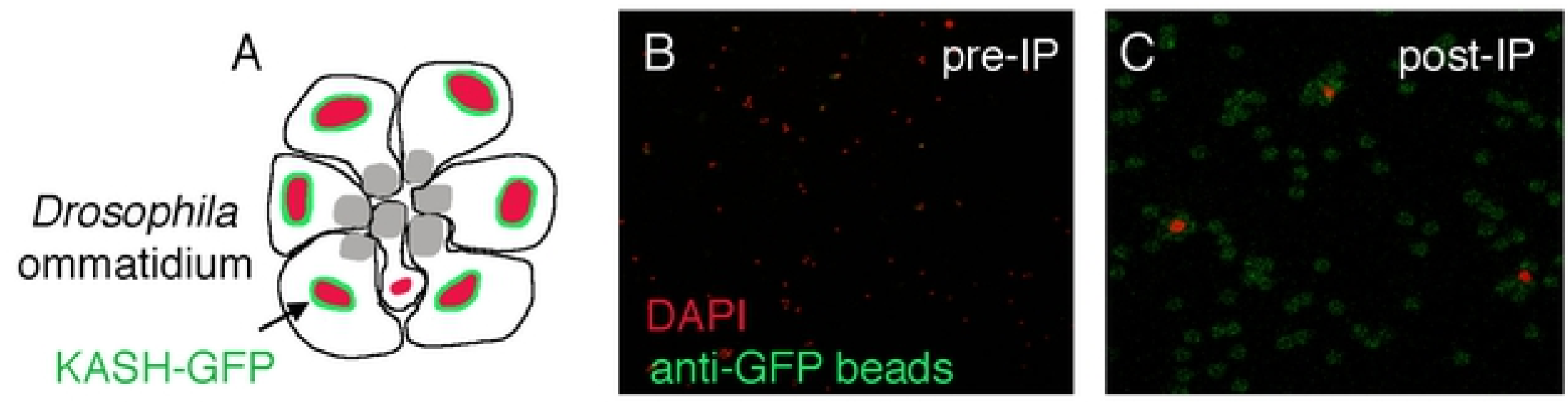

D gene expression changes

in $\operatorname{ninaE} E^{G 690} /+$

(compared to ninaE $^{\mathrm{W} T}$ )

bioRxiv preprint doi: https://doi.org/10.1101/2021.04.16.440122; this version posted April 16, 2021. The copyright holder for this preprint (which was not certified by peer review) is the author/funder, who has granted bioRxiv a license to displaycthe preprint in perpetuity. It is made
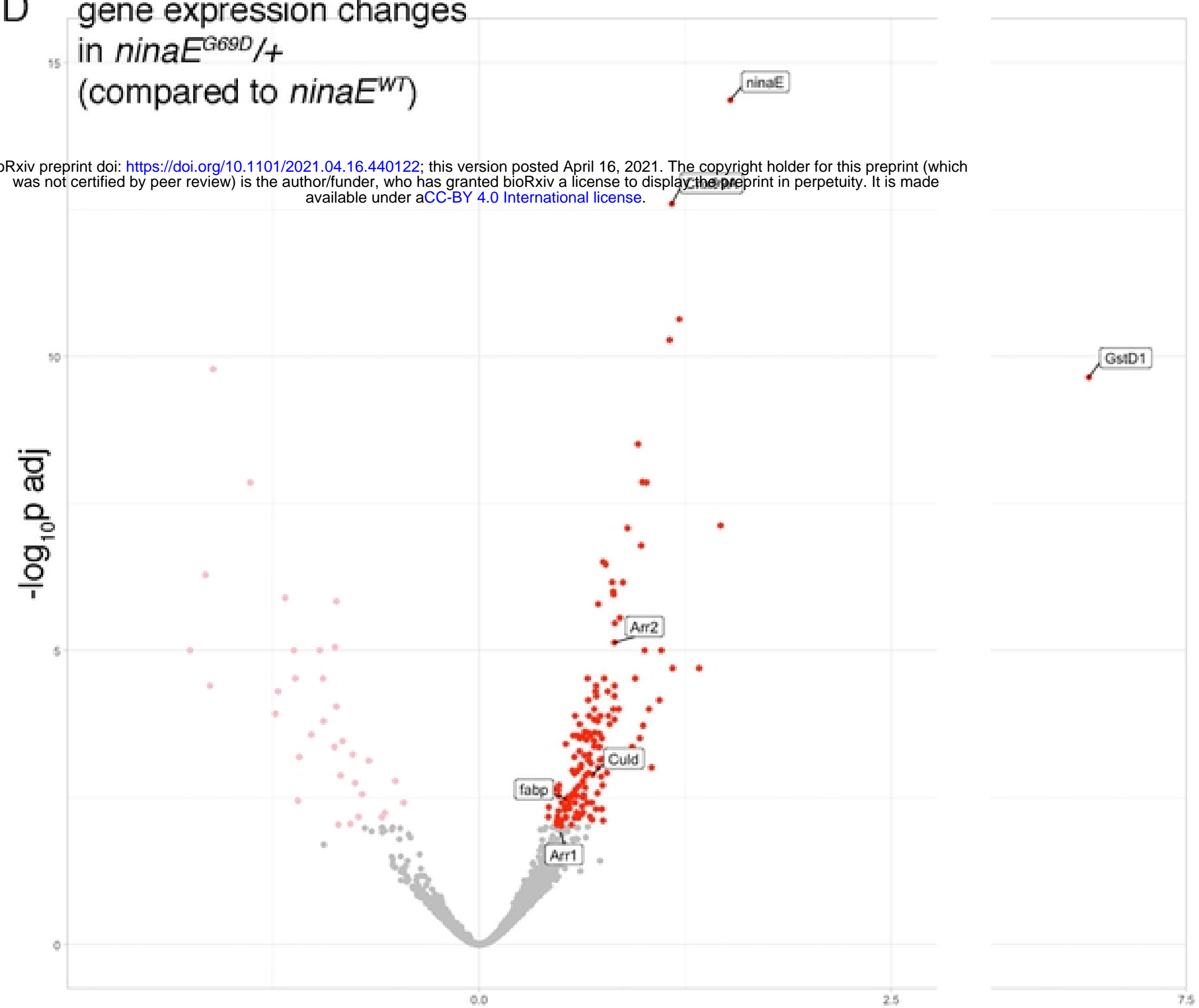

$\log _{2}$ fold change ( $n$ inaE $E^{G 69 D} /+$ vs wt)

E

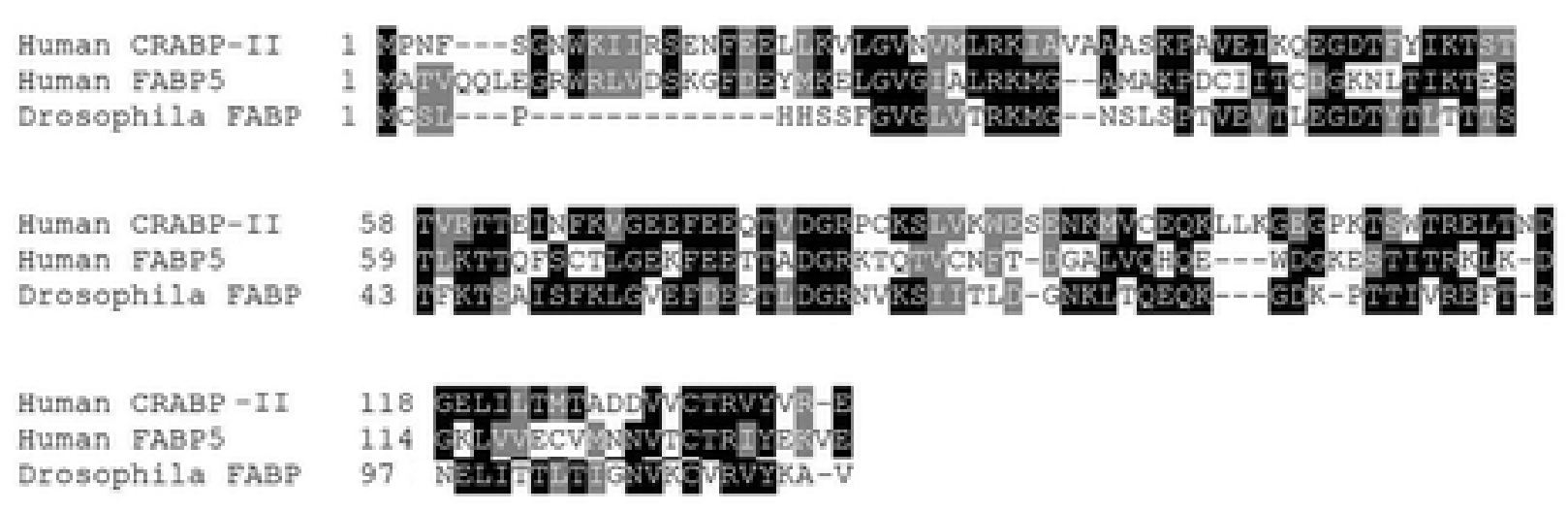

$\mathrm{F}$

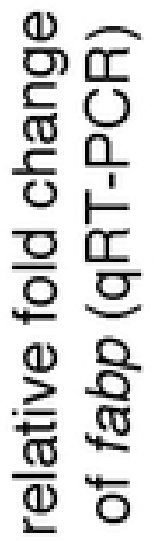

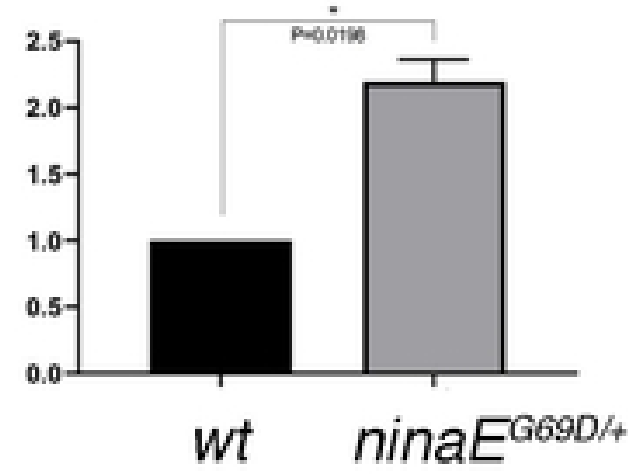

Figure 1 
A S2 cell RT-PCR

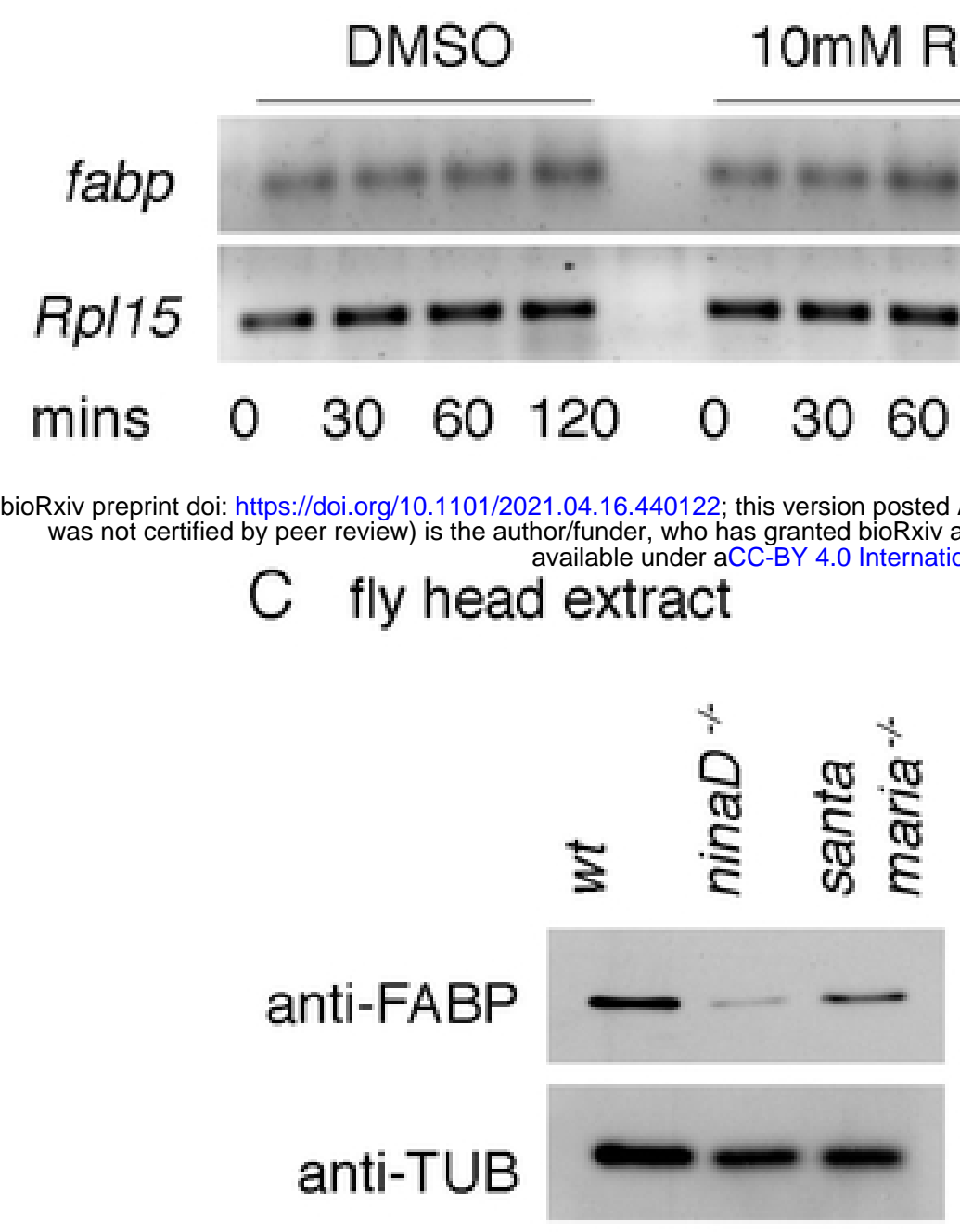

B

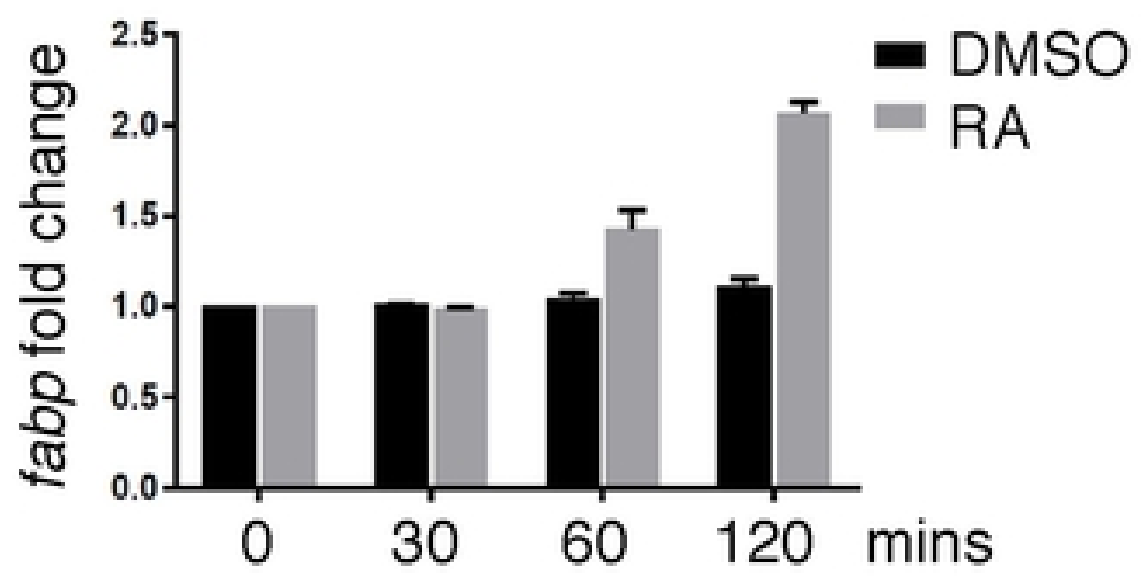

E fly head RT-PCR for Arr1 F fly head RT-PCR for Arr2

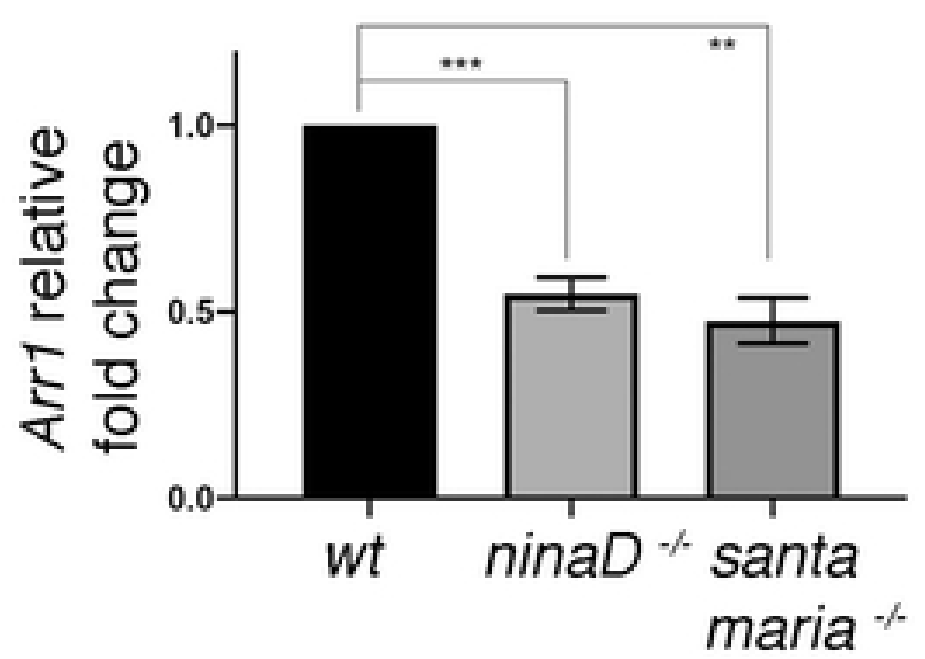

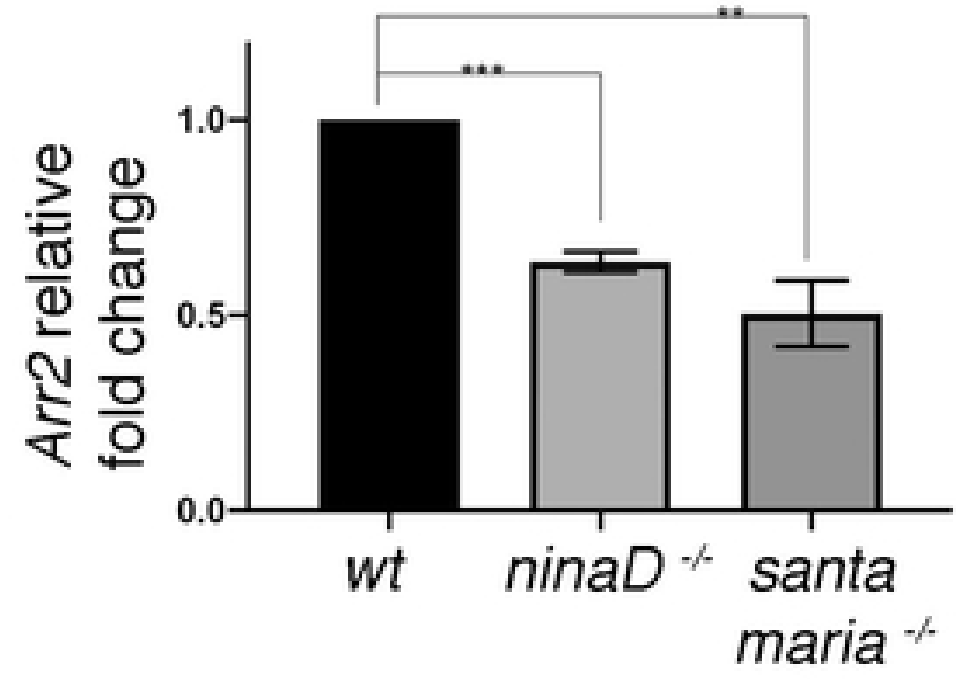

G fly head RT-PCR for fatp

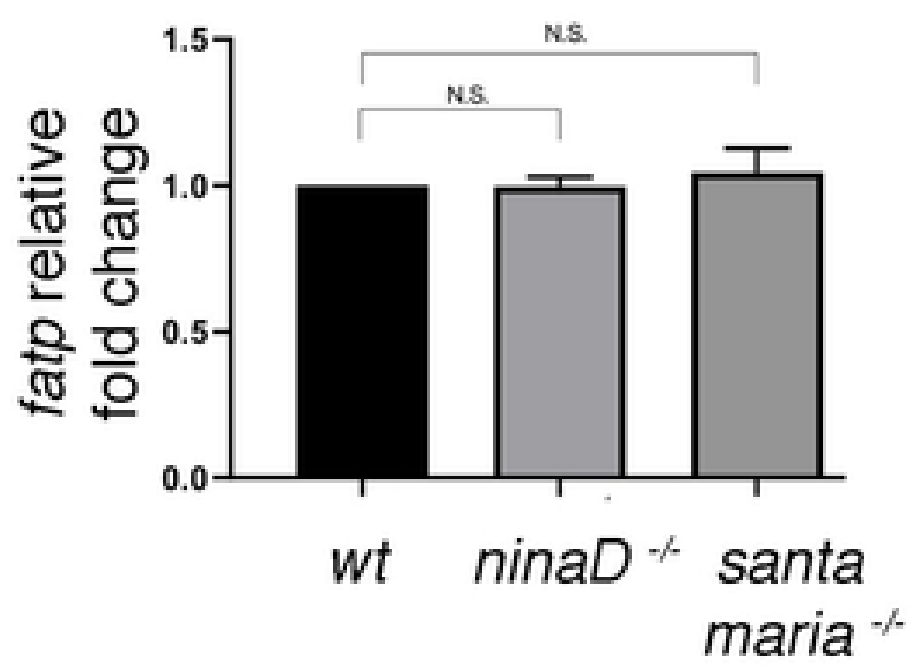

Figure 2 
A fabp locus with CA06960

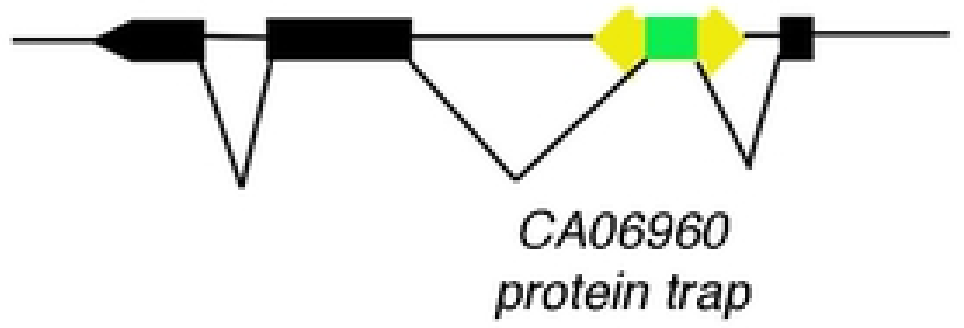

B

$\otimes 8$

bioRxiv preprint doi: https://doi.org/10.1101/201404.16. Æ 4122 ; this version posted April 16, 2021. The copyright holder was not certified by peer review) is the autankfunde(who has granted bioRxiv a license to display the preprint in pe
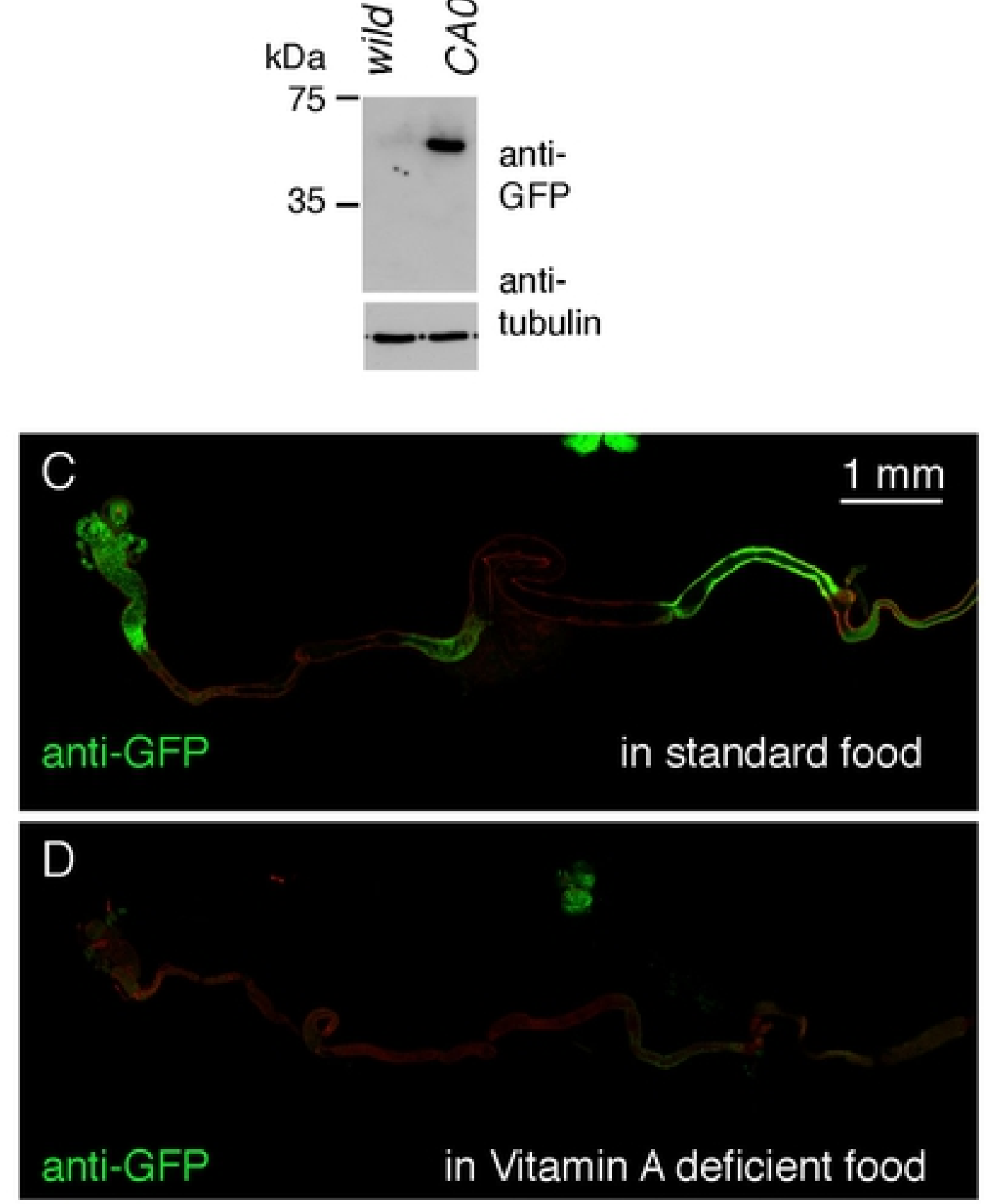

Figure 3 


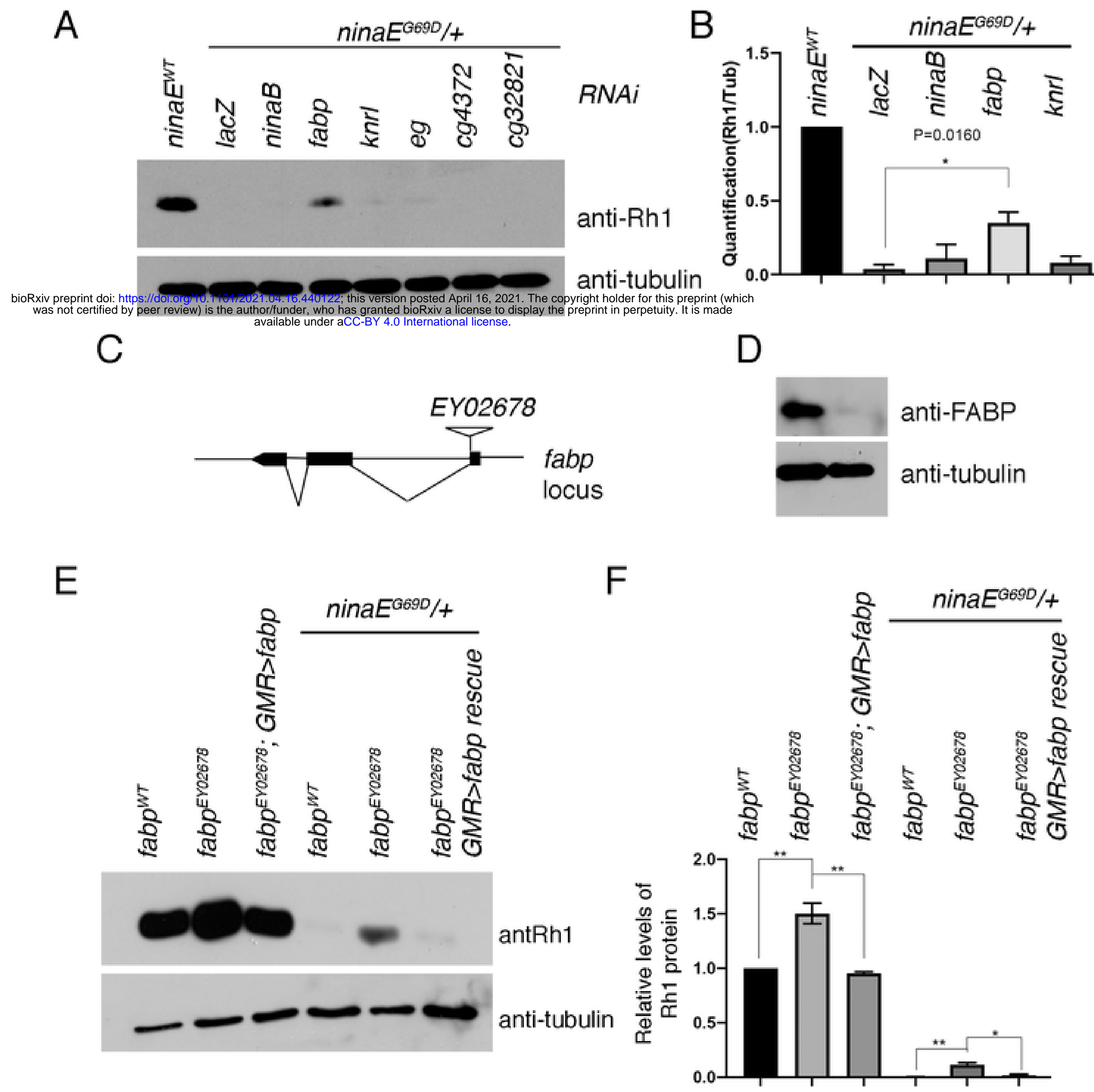

Figure 4 


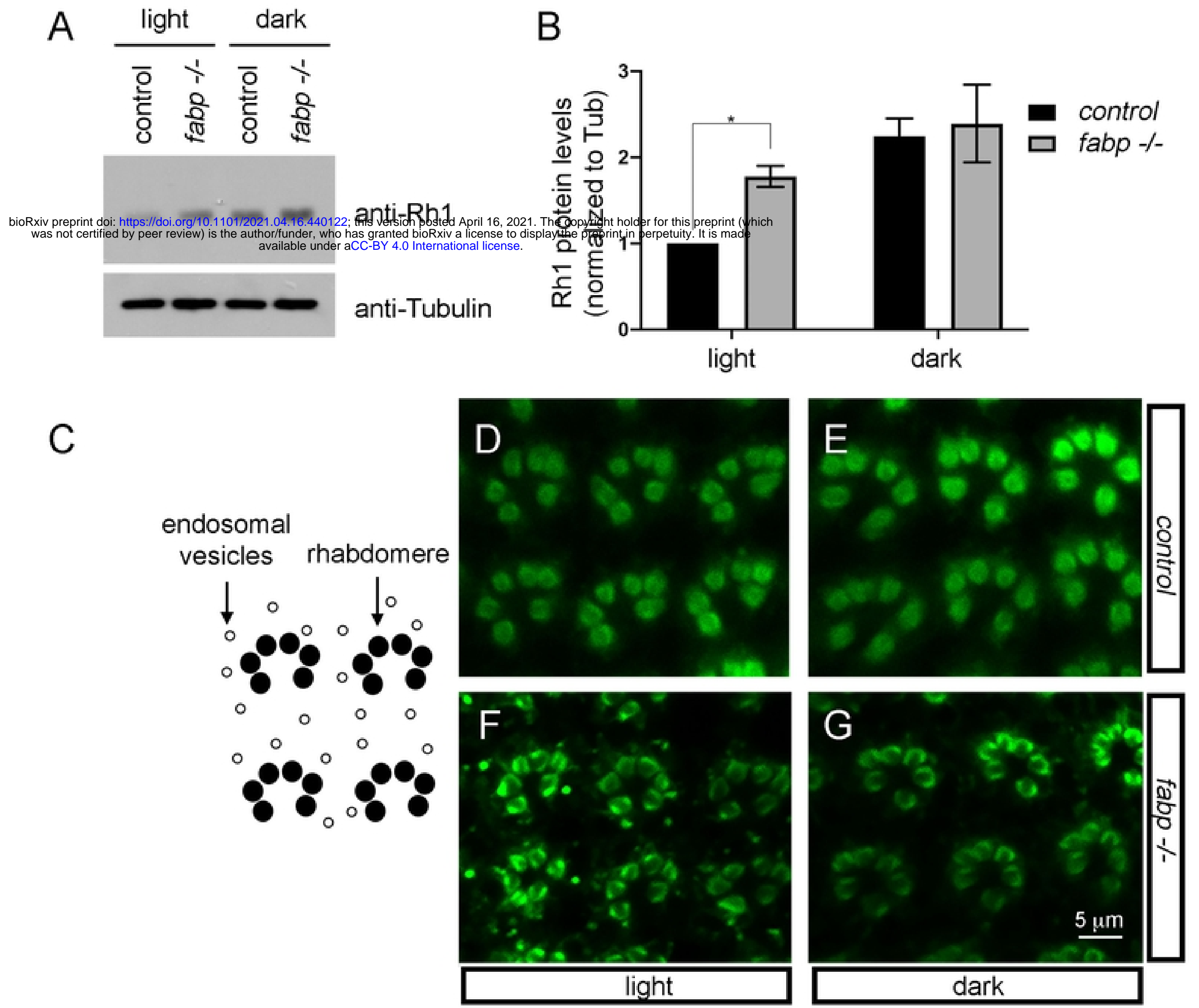

Figure 5 

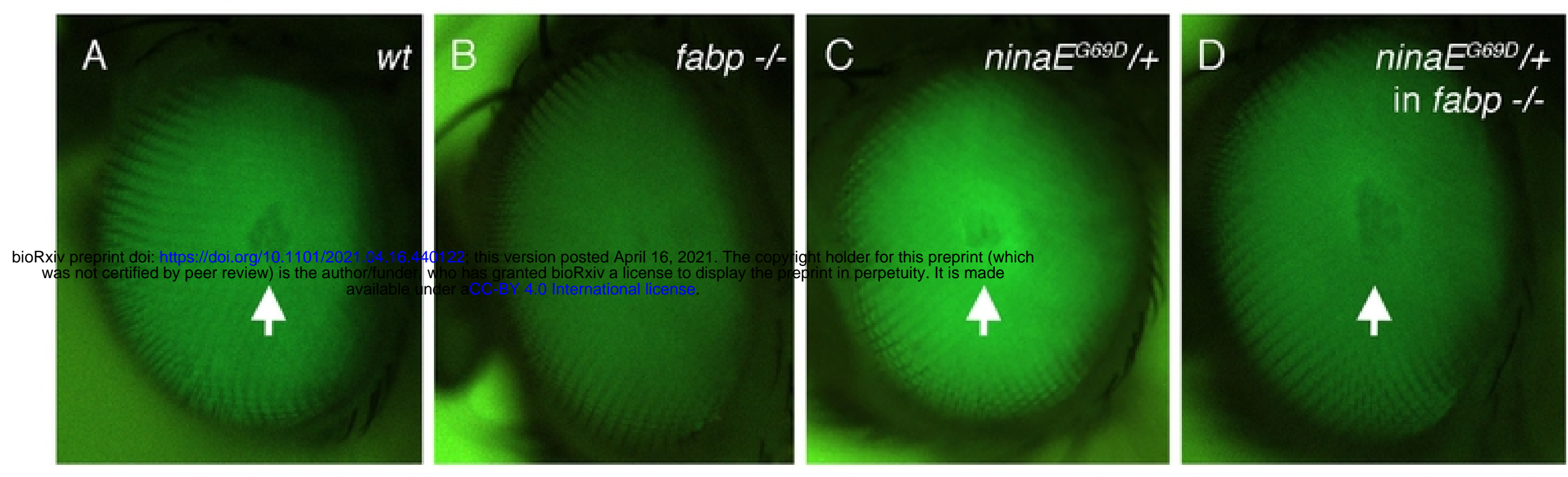

E

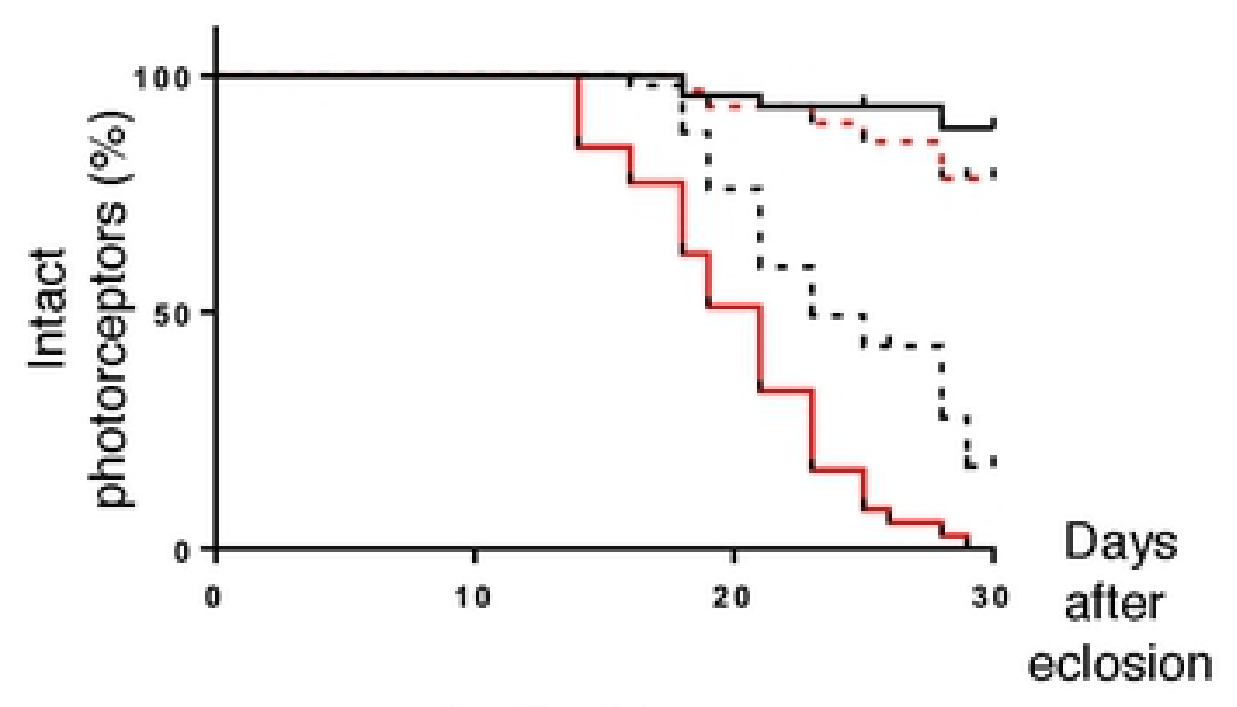

- ninaE wild type

-. $\operatorname{ninaE}^{\mathrm{G} 000 /+}$

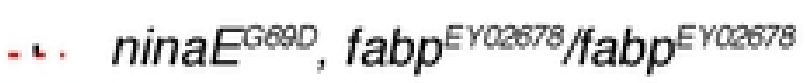

- fabp ${ }^{E Y 02678}$-/
F

Light

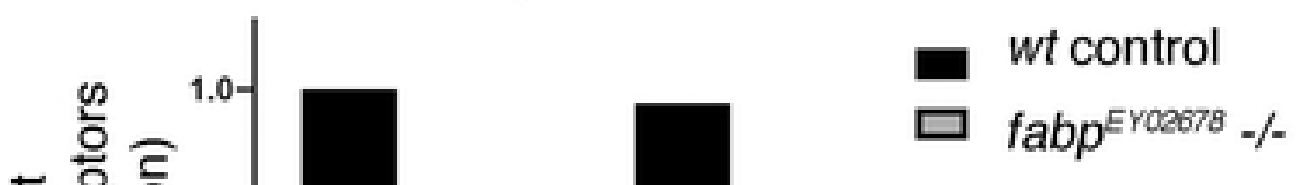

G
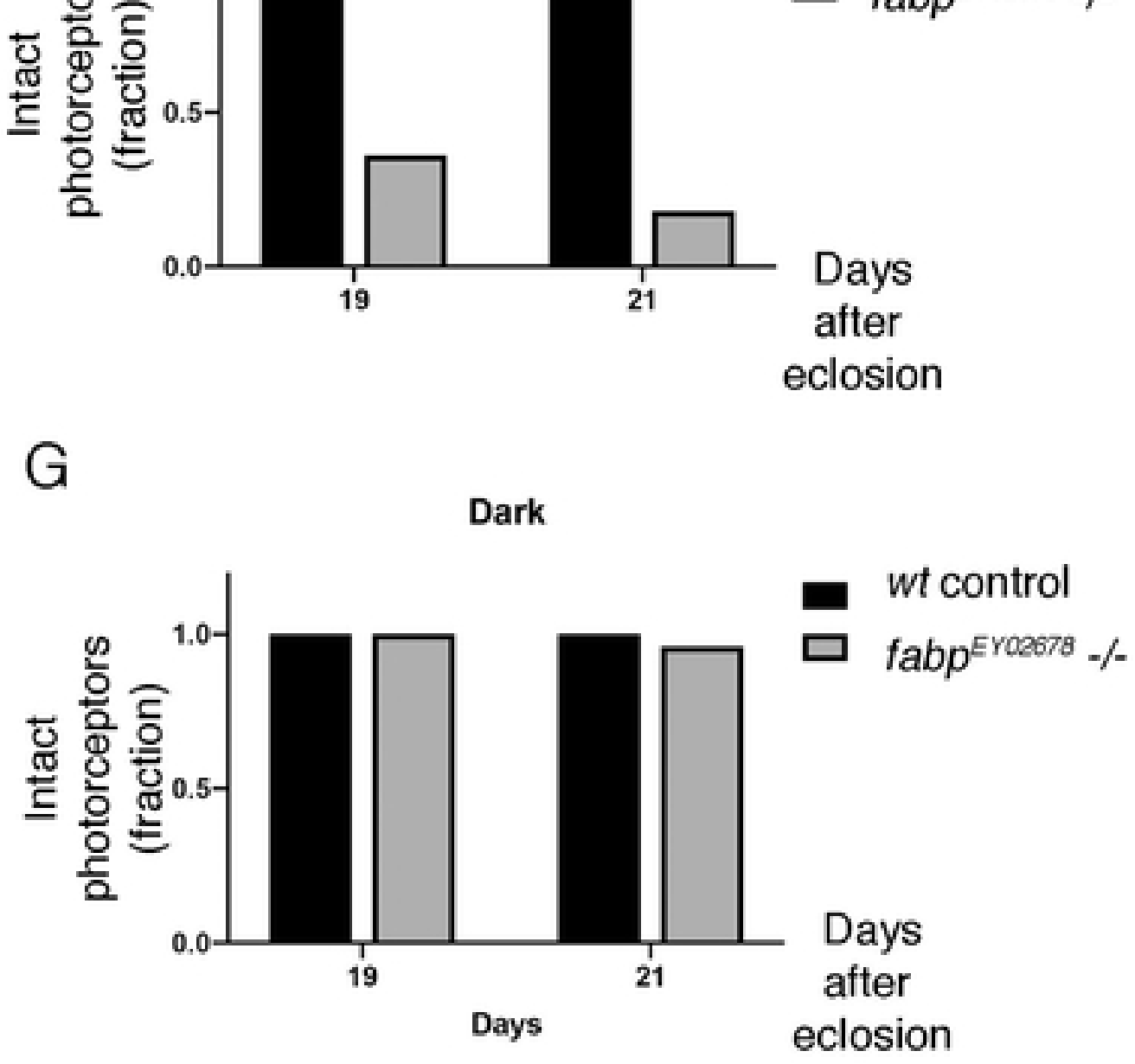

응

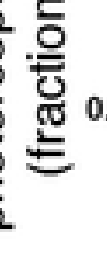

0.0

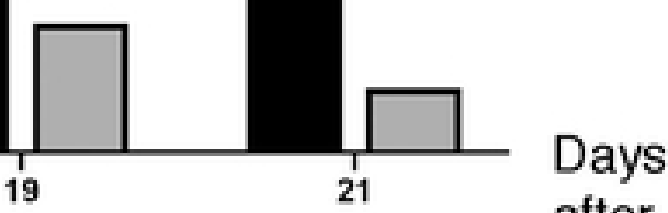

after

eclosion

Figure 6 